\title{
Prophylactic antibiotic prescribing — a study in general practice
}

\author{
A study of prophylactic antibiotic prescribing in National Health Service general dental practice in England \\ by N. A. O. Palmer, R. Pealing, R. S. Ireland and M. V. Martin BrDent J2000; 189: 43-46
}

\section{Objective}

To study the use of prophylactic antibiotics by general dental practitioners.

\section{Design}

A postal questionnaire of National Health Service (NHS) general dental practitioners in ten English Health Authorities.

\section{Subjects}

General dental practitioners (GDPs) $(1,544)$ contracted to provide NHS treatment in the Health Authorities of Liverpool, Wirral, Oxfordshire, Buckinghamshire, Nottingham, North Nottinghamshire, Sheffield, Newcastle, Northumberland and North Tyneside.

\section{Main outcome measures}

The questionnaires were analysed and the responses to each question expressed as absolute frequencies.

\section{Results}

Responses to the questionnaires were received from $929(60.1 \%)$ practitioners. Over $40 \%$ of general dental practitioners would prescribe prophylactic antibiotics for patients with no relevant medical history for minor oral surgery to prevent postoperative infection. Amoxicillin was the predominant choice of antibiotic in this situation. Between $15-67 \%$ of GDPs failed to prescribe prophylactic antibiotics for at risk medically compromised patients. GDPs also prescribed for patients with a medical history not known to be at risk from dental procedures. Over $50 \%$ of GDPs however, would seek specialist advice about prophylaxis if they were unsure of the indications and over $90 \%$ of GDPs indicated they would use the current recommended regime for antibiotic prophylaxis for patients at risk of infective endocarditis.

\section{Conclusions}

The evidence from this study suggests that a significant number of the practitioners surveyed prescribe prophylactic antibiotics inappropriately, both for surgical procedures and for patients at risk from endocarditis. There is also evidence that practitioners prescribe antibiotic prophylaxis for clinical procedures and medical conditions for which there is little evidence. The results suggest that there is a need for the development of guidelines for practitioners on the appropriate prophylactic use of antibiotics.

In brief

- Prophylactic antibiotics are being prescribed inappropriately by some general dental practitioners.

- GDPs should be conversant with current guidelines on prophylactic prescribing.

- Prophylactic antibiotics should only be used where they have been indicated as necessary.

\section{Comment}

Drophylactic antibiotics are valuable in 1 many areas of modern medicine, for example during bone marrow transplantation and to cover some forms of cardiovascular, bowel and orthopaedic surgery. The regimes employed continue to develop and in some areas of practice there is still debate about the true value of prophylactic antibiotics. Within dentistry, there are well-defined guidelines in relation to prophylaxis for patients at risk of infective endocarditis. However, in other spheres of practice, for example minor oral surgical procedures in nonmedically compromised patients, there are few guidelines and a significant amount of unnecessary prescribing of prophylactic antibiotics is believed to occur. This paper by Nick Palmer and colleagues has examined current prophylactic antibiotic prescribing, in its broadest sense, by general dental practitioners in England. Such studies are very timely in the light of the global concerns about over-use of antimicrobial drugs.

The study was based around a ques- tionnaire sent to 1,544 general dental practitioners providing NHS treatment in ten English Health Authorities. The response rate was $60.1 \%$, representing $5.9 \%$ of dentists practising within English NHS General Dental Services. While some of the findings were encouraging, such as the small proportion of respondents using prophylactic antibiotics during root canal therapy, there were some areas of concern. Thus, many respondents prescribed prophylactic antibiotics for apicectomies (43\%) and surgical extractions (39\%), despite the fact that there is no good evidence for this as a clinically useful routine practice. Conversely, only $21.8 \%$ of respondents would prescribe antibiotics to cover extractions in patients who had received head and neck radiotherapy, a situation in which prophylaxis is essential. Most practitioners were following current guidelines on antibiotic prophylaxis for patients with cardiac problems predisposing to endocarditis, though only $56 \%$ would provide cover during dental extractions for patients with ventricular septal defect. One quarter of the practitioners prescribed prophylactic antibiotics for extractions in patients with prosthetic joints, although such practice is not recommended by the British Society for Antimicrobial Chemotherapy. However, many orthopaedic surgeons recommend cover and the issue remains a vexed one for dentists who are requested by a patient's surgeon to provide prophylaxis.

This excellent study illustrates that dental surgeons would benefit from more formal guidelines on the use of prophylactic antibiotics. At present there is overprescribing in some areas, but a failure to provide adequate cover in others, with important clinical and medico-legal implications. There remains a need for high quality randomised clinical trials to develop and refine guidelines, but even based on current knowledge there is clearly room for improvement.

\section{Jeremy Bagg}

Professor of Clinical Microbiology, University of Glasgow Dental School 